\title{
Prevalência de variações anatômicas encontradas em pacientes com síndrome do túnel do carpo submetidos a liberação cirúrgica por via aberta clássica
}

\section{Prevalence of Anatomical Variations in Patients with Carpal Tunnel Syndrome Undergoing Classical Open Carpal Tunnel Release}

\author{
1 Faculdade de Medicina, Universidade de Taubaté (UNITAU), \\ Taubaté, SP, Brasil \\ ${ }^{2}$ Curso de Medicina, Faculdade de Medicina, Universidade Cidade de \\ São Paulo (UNICID), São Paulo, SP, Brasil \\ ${ }^{3}$ Departamento de Ortopedia e Traumatologia, Disciplina de Cirurgia \\ da Mão e Membro Superior, Universidade Federal de São Paulo \\ (UNIFESP), São Paulo, SP, Brasil \\ ${ }^{4}$ Serviço de Ortopedia e Traumatologia, Hospital Municipal \\ Universitário de Taubaté (H-MUT), Taubaté, SP, Brasil \\ ${ }^{5}$ Serviço de Ortopedia e Traumatologia, Hospital Regional do Vale do Paraíba \\ (HRVP), Complexo Hospitalar do Vale do Paraíba, Taubaté, SP, Brasil
}

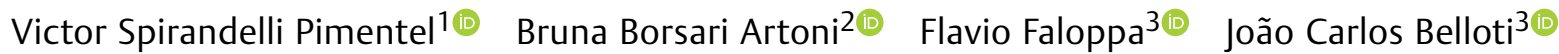

Marcel Jun Sugawara Tamaoki ${ }^{3(0)}$ Benedito Felipe Rabay Pimentel ${ }^{1,4,5(0)}$

\author{
Endereço para correspondência Benedito Felipe Rabay Pimentel, Rua \\ Francisco de Barros, 239, Centro, Taubaté, SP, 12020 230, Brasil \\ (e-mail: befecil@gmail.com).
}

Rev Bras Ortop 2022;57(4):636-641.

\author{
Resumo \\ Palavras-chave \\ - síndrome do túnel do \\ carpo \\ - nervo mediano \\ - procedimento \\ cirúrgico \\ - prevalência
}

Objetivo Avaliar a prevalência de variações anatômicas encontradas em pacientes com síndrome do túnel do carpo submetidos a liberação cirúrgica por via aberta clássica. Métodos Foram incluídos um total de 115 pacientes com alta probabilidade de diagnóstico clínico de síndrome do túnel do carpo, com indicação para o tratamento cirúrgico. Estes pacientes realizaram eletroneuromiografia e ultrassonografia para confirmação diagnóstica. Foram submetidos ao tratamento cirúrgico por via aberta clássica, no qual foi realizado um inventário completo da ferida operatória na busca e visualização de variações anatômicas intra e extra túnel do carpo.

Resultados A prevalência total das variações anatômicas intra e extra túnel do carpo encontradas neste estudo foi de $63,5 \%$ (intervalo de confiança [IC] $95 \%: 54,5-72,4 \%$ ). A prevalência do músculo transverso do carpo foi de $57,4 \%$ (IC95\%: 47,8-66,6\%), do nervo mediano bífido associado à artéria mediana persistente foi de 1,7\% (IC95\%:

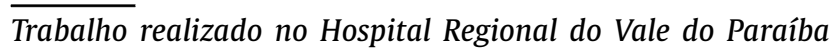
(HRVP), Complexo Hospitalar do Vale do Paraíba, Faculdade de Medicina, Universidade de Taubaté (UNITAU), Taubaté, SP, Brasil.

recebido

18 de Outubro de 2020

aceito

08 de Janeiro de 2021

Publicado on-line

Outubro 1, 2021
DOI https://doi.org/ 10.1055/s-0041-1731361. ISSN 0102-3616.
(C) 2021. Sociedade Brasileira de Ortopedia e Traumatologia. All rights reserved.

This is an open access article published by Thieme under the terms of the Creative Commons Attribution-NonDerivative-NonCommercial-License, permitting copying and reproduction so long as the original work is given appropriate credit. Contents may not be used for commercial purposes, or adapted, remixed, transformed or built upon. (https://creativecommons.org/ licenses/by-nc-nd/4.0/)

Thieme Revinter Publicações Ltda., Rua do Matoso 170, Rio de Janeiro, RJ, CEP 20270-135, Brazil 


\section{Abstract}

\section{Keywords}

- carpal tunnel syndrome

- median nerve

- surgical procedures

- prevalence
0,0-4,2\%) e do nervo mediano bífido associado à artéria mediana persistente e ao músculo transverso do carpo foi de 1,7\% (IC95\%: 0,0-4,2\%).

Conclusão A variação anatômica extra túnel do carpo mais prevalente foi o músculo transverso do carpo e a variação anatômica intra túnel do carpo mais prevalente foi o nervo mediano bífido associado à artéria mediana persistente. O achado cirúrgico de uma variação anatômica extra túnel do carpo, como o músculo transverso do carpo, pode nos indicar a presença de outras variações anatômicas intra túnel do carpo associadas, como nervo mediano bífido, artéria mediana persistente e variações anatômicas do ramo recorrente do nervo mediano.

Objective To evaluate the prevalence of anatomical variations encountered in patients with carpal tunnel syndrome who underwent carpal tunnel classical open release.

Methods A total of 115 patients with a high probability of clinical diagnosis for carpal tunnel syndrome and indication for surgical treatment were included. These patients underwent electroneuromyography and ultrasound for diagnostic confirmation. They underwent surgical treatment by carpal tunnel classical open release, in which a complete inventory of the surgical wound was performed in the search and visualization of anatomical variations intra- and extra-carpal tunnel.

Results The total prevalence of anatomical variations intra- and extra-carpal tunnel found in this study was $63.5 \%$ (95\% confidence interval $[\mathrm{Cl}]$ : $54.5-72.4)$. The prevalence of the carpal transverse muscle was $57.4 \%$ (95\% Cl: $47.8-66.6 \%)$, of the bifid median nerve associated with the persistent median artery was $1.7 \%(95 \% \mathrm{Cl}: 0.0-4.2 \%)$, and the median bifid nerve associated with the persistent median artery and the transverse carpal muscle was $1.7 \%$ (95\% Cl: $0.0-4.2 \%)$.

Conclusion The most prevalent extra-carpal tunnel anatomical variation was carpal transverse muscle. The most prevalent intra-carpal tunnel anatomical variation was median bifid nerve associated with the persistent median artery. The surgical finding of an extra-carpal tunnel anatomical variation, such as the transverse carpal muscle, may indicate the presence of other associated carpal intra tunnel anatomical variations, such as the bifid median nerve, persistent median artery, and anatomical variations of the recurrent median nerve branch.

\section{Introdução}

A síndrome do túnel do carpo (STC), caracterizada pela compressão do nervo mediano ao nível do punho, é a neuropatia compressiva mais comum do membro superior. ${ }^{1}$ O seu tratamento cirúrgico com a liberação aberta clássica do túnel do carpo é um dos procedimentos eletivos mais realizados em cirurgia da mão. ${ }^{1,2}$ Um planejamento pré-operatório adequado, um conhecimento confiável da anatomia e um entendimento das diferenças anatômicas inesperadas durante a cirurgia são requisitos indispensáveis para a prevenção de desfechos cirúrgicos desfavoráveis. Principalmente em uma região em que as variações anatômicas intra e extra túnel do carpo são frequentes, com valores de prevalência amplamente variáveis, de acordo com o desenho do estudo. ${ }^{3,4}$ Além disto, a associação destas variações anatômicas entre si e a sua relação com a STC são questões ainda divergentes e parcialmente entendidas na literatura. Estudos primários bem desenhados de boa qualidade metodológica de prevalência de variações anatômicas em pacientes com
STC são incomuns. ${ }^{3,4}$ Por isso, foi proposto um desenho de estudo baseado na prática clínica de rotina do especialista na STC, com o objetivo de avaliar a prevalência de variações anatômicas encontradas em pacientes com STC submetidos a liberação cirúrgica por via aberta clássica do túnel do carpo.

\section{Materiais e métodos}

Trata-se de um estudo de prevalência, primário, transversal e de centro único, aprovado pelo comitê de ética em pesquisa de nossa instituição sob o número 11/19. Foram incluídos um total de 115 pacientes $(n=115)$, todos do gênero feminino, com idade entre 40 e 80 anos, com acometimento unilateral ou bilateral, que concordaram em participar do estudo e assinaram o termo de consentimento livre e esclarecido, e que foram submetidos a tratamento conservador prévio sem melhora clínica efetiva e com um escore de probabilidade de diagnóstico clínico de STC igual ou superior a 12 pontos, de acordo com a lista de seis critérios para o diagnóstico de STC 
(CTS-6, na sigla em inglês) de Graham et al. ${ }^{5}$ A CTS-6 foi utilizada na avaliação clínica dos pacientes potencialmente elegíveis deste estudo, e suas respectivas pontuações foram: parestesia (3,5), parestesia noturna $(4,0)$, hipotrofia e ou atrofia da musculatura tenar $(5,0)$, teste de Tinel $(4,0)$, teste de Phalen $(5,0)$ e teste estático da discriminação dos 2 pontos $(5,0)$. Foram excluídos desta amostra os pacientes com radiculopatia cervical, outras síndromes compressivas dos membros superiores, polineuropatia, com história de liberação cirúrgica prévia do túnel do carpo e aqueles com sequelas de fratura do punho.

Após a avaliação clínica, os pacientes elegíveis deste estudo foram encaminhados para exames complementares. Um valor $\geq 10,0 \mathrm{~mm}^{2}$ da área de secção transversa no limite anatômico proximal do túnel do carpo pelo método direto de mensuração pela ultrassonografia do punho, e uma velocidade de condução sensitiva do nervo mediano inferior a $50 \mathrm{~m} / \mathrm{s}$ associado a uma latência motora distal do nervo mediano $\geq 4,2$ milissegundos pela eletroneuromiografia dos membros superiores, confirmaram o diagnóstico de STC. ${ }^{6-11}$ Na sequência, os pacientes foram encaminhados e submetidos ao tratamento cirúrgico de caráter ambulatorial,

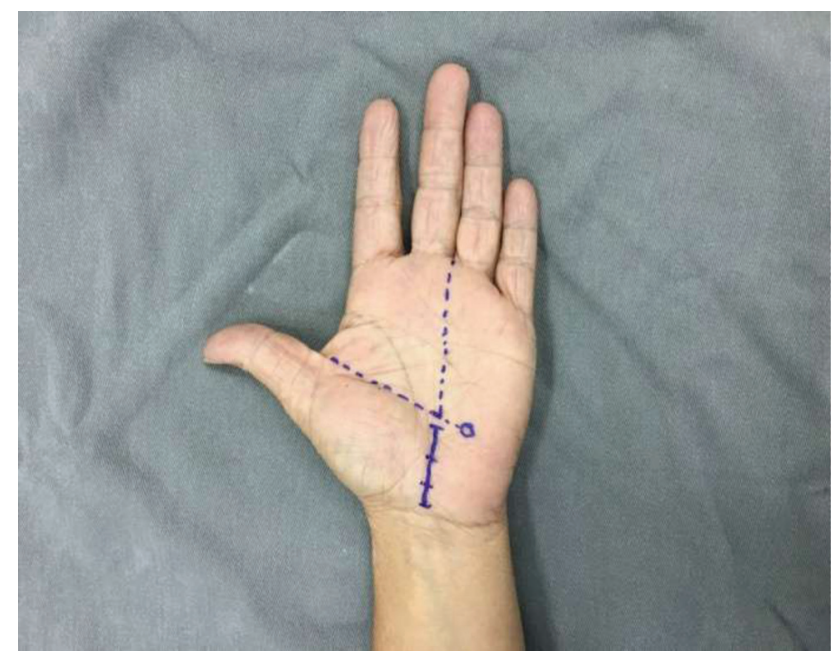

Fig. 1 Planejamento da incisão cirúrgica pela técnica de liberação aberta clássica do túnel do carpo.

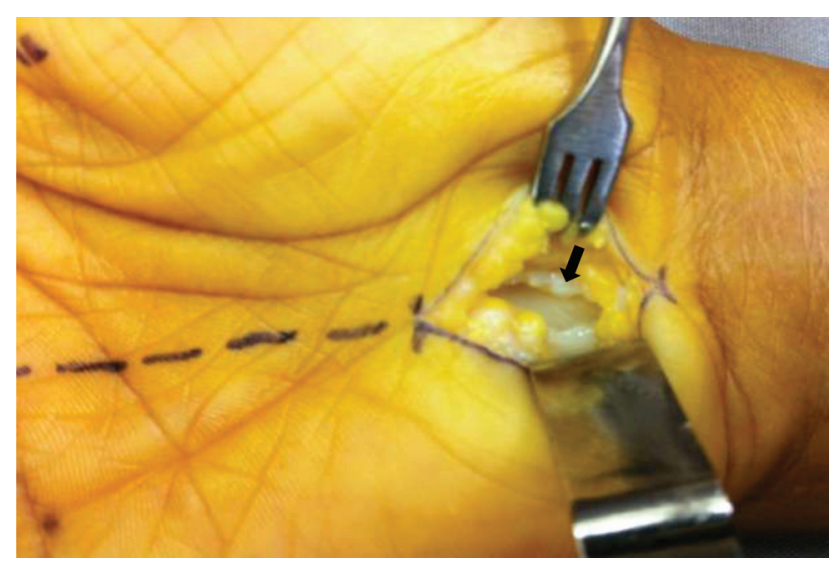

Fig. 2 Abordagem cirúrgica mostrando o ligamento transverso do carpo (seta) completamente aberto. realizado em centro cirúrgico de forma consecutiva e por um mesmo cirurgião da mão. Na sala cirúrgica, os pacientes foram posicionados na mesa cirúrgica em decúbito dorsal horizontal com a mão e o punho apoiados em uma mesa auxiliar. ${ }^{12}$ Foram submetidos à técnica de anestesia regional endovenosa de Bier no membro acometido. ${ }^{13} \mathrm{~A}$ técnica cirúrgica utilizada foi a liberação por via aberta clássica do túnel do carpo, através de uma incisão longitudinal palmar de cerca de $2 \mathrm{~cm}$ de comprimento que se alinhava com o terceiro espaço interdigital da mão, mas não se estendia proximalmente além da prega de flexão distal do punho e, distalmente, além da linha de Kaplan (-Figura 1). A abordagem cirúrgica foi realizada por planos anatômicos: pele, tecido celular subcutâneo, fáscia palmar, e ligamento transverso do carpo, sendo este aberto completamente em direção distal e proximal ao túnel do carpo, liberando amplamente o nervo mediano (- Figura 2). ${ }^{12-14}$

Durante a cirurgia, foi realizado um inventário completo da ferida operatória na busca e visualização de variações anatômicas intra e extra túnel do carpo (-Figuras 3 e 4).

Após o tratamento cirúrgico, os pacientes receberam alta médica no mesmo dia e foram acompanhados

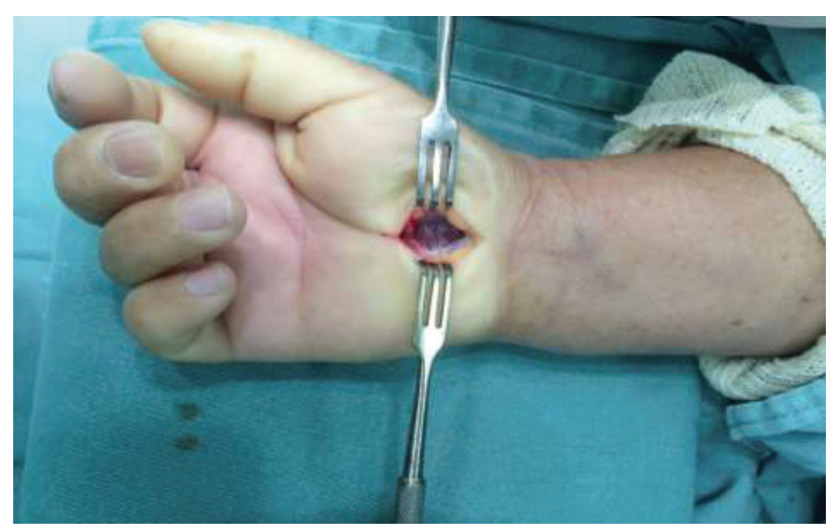

Fig. 3 Achado cirúrgico do músculo transverso do carpo.

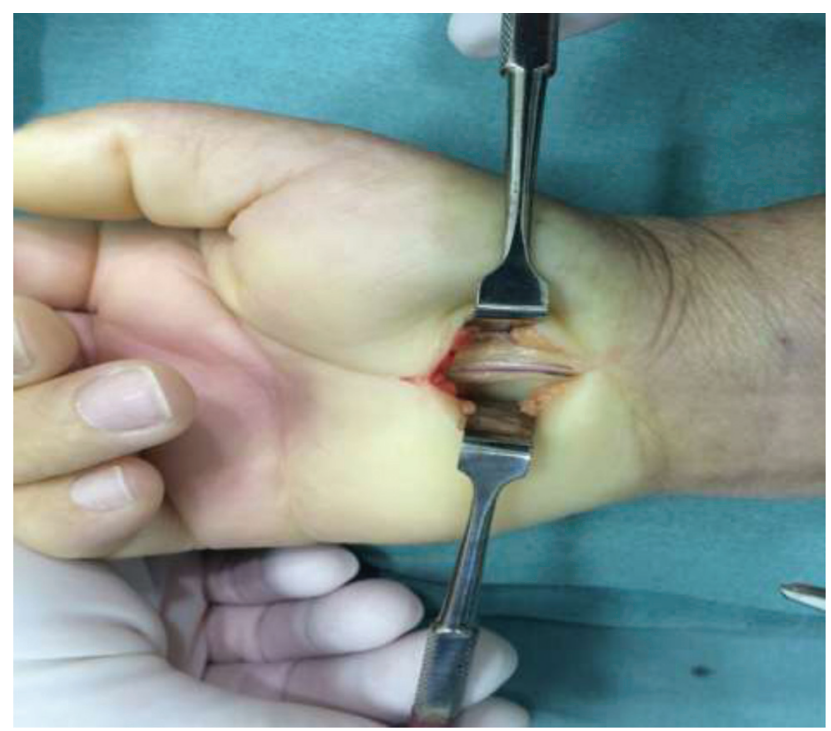

Fig. 4 Achado cirúrgico do nervo mediano bífido associado à artéria mediana persistente. 


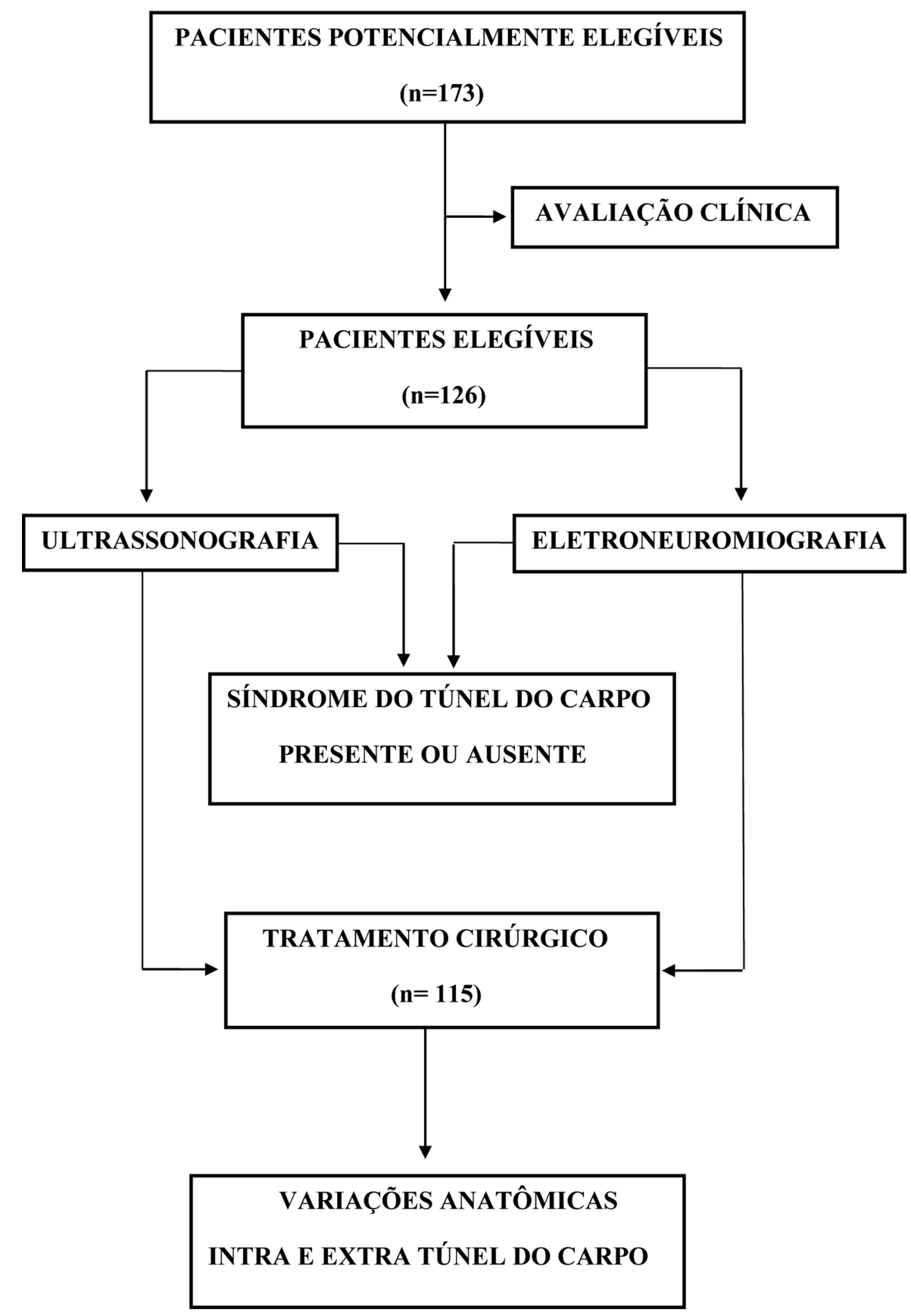

Fig. 5 Fluxograma do estudo.

ambulatorialmente por um período de 6 meses. Foram recrutados $10 \%$ a mais do total de pacientes elegíveis para cobrir possíveis perdas ou exclusões no decorrer do estudo. A - Figura 5 mostra cada etapa deste estudo.

\section{Análise estatística}

As variáveis categóricas foram apresentadas como frequências relativas e absolutas e as variáveis numéricas foram apresentadas como medidas-resumo. ${ }^{15}$ A prevalência das variações anatômicas intra e extra túnel do carpo encontradas neste estudo foram apresentadas como frequências relativas e absolutas, bem como seus respectivos intervalos de confiança de $95 \%$ e foram calculadas através do software estatístico STATA 12 (Stata Corp. LLC, College Station, TX, EUA).

\section{Resultados}

\section{Características dos pacientes}

Avaliou-se um total de 115 pacientes. A média das idades foi de 52,9 anos (desvio padrão=9,1 anos), com uma idade mínima de 40 e máxima de 79 anos. A idade mediana foi de 52 anos. Com relação ao tempo da doença, a média observada foi de 4,0 anos (desvio padrão=3,2 anos), com um mínimo de 1 ano e máximo de 20 anos. A mediana foi de 3 anos de tempo de doença. Quanto à lateralidade, 109 pacientes 
Tabela 1 Prevalência de variações anatômicas encontradas em pacientes com síndrome do túnel do carpo submetidos a liberação cirúrgica por via aberta clássica do túnel do carpo

\begin{tabular}{|l|l|l|}
\hline Variações anatômicas & $\mathbf{N}(\%)$ & IC95\% (\%) \\
\hline MTC & $66(57,4)$ & $47,8-66,6$ \\
\hline NMB + AMP & $2(1,7)$ & $0,0-4,2$ \\
\hline NMB + AMP + MTC & $2(1,7)$ & $0,0-4,2$ \\
\hline MTC + RRT & $1(0,9)$ & $0,0-2,6$ \\
\hline MTC + VMF & $1(0,9)$ & $0,0-2,6$ \\
\hline RRS & $1(0,9)$ & 0,0 a 2,6 \\
\hline TOTAL & $73(63,5)$ & $54,5-72,4$ \\
\hline
\end{tabular}

Abreviaturas: AMP, artéria mediana persistente; IC95\%, intervalo de confiança de $95 \%$ para as proporções; MTC, músculo transverso do carpo; NMB, nervo mediano bífido; RRS ramo recorrente subligamentar do nervo mediano; RRT, ramo recorrente transligamentar do nervo mediano; VMF, ventre muscular do tendão flexor superficial dedo longo. $\mathrm{n}=115$.

Os resultados são apresentados como a porcentagem total.

$(90,8 \%)$ apresentaram STC bilateral e 6 pacientes $(5,2 \%)$ apresentaram STC unilateral. Em 59 pacientes (51,3\%), o lado acometido foi a mão direita, e em 56 pacientes $(48,7 \%)$ foi a mão esquerda. Dos 73 pacientes $(63,5 \%)$ que apresentaram variações anatômicas intra e extra túnel do carpo, 38 pacientes $(52,0 \%)$ operaram a mão direita e 35 pacientes $(48,0 \%)$ operaram a mão esquerda. A mão dominante era à direita em 65 pacientes $(89,0 \%)$ e a esquerda em 8 pacientes $(11,0 \%)$.

\section{Prevalência de variações anatômicas}

A prevalência total de variações anatômicas encontradas nos pacientes deste estudo foi de 63,5\% (IC95\%: 54,5-72,4\%). Em relação às variações anatômicas extra túnel do carpo, a prevalência do músculo transverso do carpo foi de 57,4\% (intervalo de confiança [IC]95\%: 47,8-66,6\%). Quanto às variações anatômicas intra túnel do carpo, a prevalência do nervo mediano bífido associado à artéria mediana persistente foi de 1,7\% (IC95\%: 0,0-4,2\%). Quanto às variações intra e extra túnel do carpo associadas, a prevalência do nervo mediano bífido associado à artéria mediana persistente e ao músculo transverso do carpo foi de 1,7\% (IC95\%: 0,0-4,2\%). Outras variações anatômicas associadas encontradas foram músculo transverso do carpo associado ao ramo recorrente do nervo mediano transligamentar, músculo transverso do carpo associado a um ventre muscular do tendão flexor superficial do dedo longo e ramo recorrente do nervo mediano subligamentar. Cada uma destas apresentou uma prevalência de 0,9\% (IC95\%: 0,0-2,6\%). A - Tabela 1 mostra um resumo dos resultados deste estudo.

\section{Discussão}

Este estudo segue uma linha de pesquisa na STC em nossa instituição e originou-se de um ensaio clínico de acurácia baseado na efetividade da ultrassonografia e dos estudos de condução nervosa no diagnóstico da STC. ${ }^{16}$ As variações anatômicas do túnel do carpo foram classificadas por Singer e Asworth ${ }^{17}$ em tipo I, intrínsecas ou intra túnel do carpo, quando encontradas dentro do túnel do carpo, e tipo II, extrínsecas ou extra túnel do carpo, quando recobrem o ligamento transverso do carpo. Tanto as variações anatômicas intra como extra túnel do carpo, associadas ou não, encontradas em nosso estudo estavam diretamente relacionadas à probabilidade de diagnóstico clínico da STC. ${ }^{18,19}$ De acordo com Green e Morgan, ${ }^{20}$ na presença de uma variação anatômica extra túnel do carpo como o músculo transverso do carpo, há uma probabilidade superior a $90 \%$ de que o ramo recorrente do nervo mediano seja anômalo, como o ramo transligamentar encontrado em nosso estudo. A faixa etária mais jovem e a mão dominante dos pacientes com STC em nosso estudo não foram fatores determinantes para correlacionar a uma maior prevalência de variações anatômicas intra e extra túnel do carpo como foi observado no estudo de Singer e Asworth. ${ }^{17}$ O nervo mediano bífido é uma variação anatômica intra túnel do carpo representada por uma bifurcação alta do nervo mediano, proximal ao túnel do carpo. $^{21,22}$ Esta variação anatômica em $45 \%$ dos casos, pode coexistir com uma artéria mediana persistente. ${ }^{19,22,23}$ Os valores de prevalência do nervo mediano bífido associado à artéria mediana persistente e da prevalência total das variações anatômicas intra túnel do carpo encontradas em nosso estudo foram similares aos valores encontrados no estudo cirúrgico de Lindley and Kleinert ${ }^{3} \mathrm{O}$ músculo transverso do carpo é uma variação anatômica extra túnel do carpo descrita por Rangoowansi et al. ${ }^{24}$ como fibras musculares transversas que recobrem ou se interpõem ao ligamento transverso do carpo. Em nosso estudo, o valor da prevalência do músculo transverso do carpo foi acima do esperado, principalmente quando comparamos com os valores da prevalência de estudos da literatura cirúrgica $^{20,21,24-27}$ Tuncalli et al. ${ }^{26}$ já nos alertava sobre isto e afirmava que o músculo transverso do carpo era descrito de forma semelhante na maioria dos estudos, mas também era interpretado de forma diferente por vários autores. Acreditava que, a frequência do músculo transverso do carpo era substimada na literatura, sendo mais prevalente do que se pensava, pois quando era encontrado em cirurgia de liberação aberta do túnel do carpo era erroneamente interpretado como uma hipertrofia da musculatura tenar ou como o músculo palmar curto ou ainda devido à uma incisão cirúrgica mais radial, não alinhada com o terceiro espaço interdigital da mão, não estando de acordo com a técnica cirúrgica. ${ }^{26}$ A principal limitação deste estudo foi a ausência de um grupo de estudo em cadáveres das variações anatômicas intra e extra túnel do carpo encontradas em nosso estudo cirúrgico. $O$ ponto forte deste estudo foi seu delineamento e sua consistência utilizando-se de uma amostra de pacientes com alta probabilidade diagnóstica de STC, que realizaram um bom planejamento pré-operatório e foram submetidos uma técnica cirúrgica universal e reprodutível. Como perspectiva futura, seria interessante realizar um estudo que mostre como se comportam os achados clínicos e complementares dos pacientes com STC na presença destas variações anatômicas intra e extra túnel do carpo. 


\section{Conclusões}

Em pacientes com síndrome do túnel do carpo submetidos a liberação cirúrgica por via aberta clássica, a variação anatômica extra túnel do carpo mais prevalente foi o músculo transverso do carpo e a variação anatômica intra túnel do carpo mais prevalente foi o nervo mediano bífido associado à artéria mediana persistente.

O achado cirúrgico de uma variação anatômica extra túnel do carpo, como o músculo transverso do carpo, pode nos revelar a presença de outras variações anatômicas intra túnel do carpo associadas, como nervo mediano bífido, artéria mediana persistente e variações anatômicas do ramo recorrente do nervo mediano.

\section{Suporte Financeiro}

Não houve suporte financeiro de fontes públicas, comerciais, ou sem fins lucrativos.

Conflito de interesses

Os autores declaram não haver conflito de interesses.

\section{Referências}

1 Bickel KD. Carpal tunnel syndrome. J Hand Surg Am 2010;35(01): 147-152

2 Afshar A, Nasiri B, Mousavi SA, Hesarikia H, Navaeifar N, Taleb H. Anatomic Anomalies Encountered in 467 Open Carpal Tunnel Surgeries. Arch Iran Med 2016;19(04):285-287

3 Lindley SG, Kleinert JM. Prevalence of anatomic variations encountered in elective carpal tunnel release. J Hand Surg Am 2003;28(05):849-855

4 Walker FO, Cartwright MS, Blocker JN, et al. Prevalence of bifid median nerves and persistent median arteries and their association with carpal tunnel syndrome in a sample of Latino poultry processors and other manual workers [published correction appears in Muscle Nerve. 2014 Feb;49(2):297. Schultz, Mark R [corrected to Schulz, Mark R]]. Muscle Nerve 2013;48(04):539-544

5 Graham B, Regehr G, Naglie G, Wright JG. Development and validation of diagnostic criteria for carpal tunnel syndrome. J Hand Surg Am 2006;31(06):919-924

6 Trachani E, Rigopoulou A, Veltsista D, Gavanozi E, Chrysanthopoulou A, Chroni E. Occurrence of bifid median nerve in healthy and carpal tunnel syndrome patients. J Electromyogr Kinesiol 2018;39:77-80

7 Pimentel BF, Abicalaf CA, Braga L, et al. Cross-sectional area of the median nerve characterized by ultrasound in patients with carpal tunnel syndrome before and after the release of the transverse carpal ligament. J Diagn Med Sonogr 2013;29(03):116-121

8 Granata G, Caliandro P, Pazzaglia C, et al. Prevalence of bifid median nerve at wrist assessed through ultrasound. Neurol Sci 2011;32(04):615-618

9 Duncan I, Sullivan P, Lomas F. Sonography in the diagnosis of carpal tunnel syndrome. AJR Am J Roentgenol 1999;173(03):681-684
10 Ntani G, Palmer KT, Linaker C, et al. Symptoms, signs and nerve conduction velocities in patients with suspected carpal tunnel syndrome. BMC Musculoskelet Disord 2013;14:242

11 Jablecki CK, Andary MT, So YT, Wilkins DE, Williams FHAAEM Quality Assurance Committee. Literature review of the usefulness of nerve conduction studies and electromyography for the evaluation of patients with carpal tunnel syndrome. Muscle Nerve 1993;16(12):1392-1414

12 Louie D, Earp B, Blazar P. Long-term outcomes of carpal tunnel release: a critical review of the literature. Hand (N Y) 2012;7(03): 242-246

13 Brill S, Middleton W, Brill G, Fisher A. Bier's block; 100 years old and still going strong!. Acta Anaesthesiol Scand 2004;48(01): 117-122

14 Rodner CM, Katarinic J. Open carpal tunnel release. Tech Orthop 2006;21(01):3-11

15 Machin D, Campbell MJ, Tan SB, Tan SH. Sample Size Tables for Clinical Studies. 3rd ed. United Kingdom: Willey-Blackwell; 2009

16 Pimentel BFR, Faloppa F, Tamaoki MJS, Belloti JC. Effectiveness of ultrasonography and nerve conduction studies in the diagnosing of carpal tunnel syndrome: clinical trial on accuracy. BMC Musculoskelet Disord 2018;19(01):115

17 Singer G, Ashworth CR. Anatomic variations and carpal tunnel syndrome: 10-year clinical experience. Clin Orthop Relat Res 2001;(392):330-340

18 Prime MS, Palmer J, Khan WS, Goddard NJ. Is there Light at the End of the Tunnel? Controversies in the Diagnosis and Management of Carpal Tunnel Syndrome. Hand (N Y) 2010;5(04): 354-360

19 Bayrak IK, Bayrak AO, Kale M, Turker H, Diren B. Bifid median nerve in patients with carpal tunnel syndrome. J Ultrasound Med 2008;27(08):1129-1136

20 Green DP, Morgan JP. Correlation between muscle morphology of the transverse carpal ligament and branching pattern of the motor branch of median nerve. J Hand Surg Am 2008;33(09): 1505-1511

21 Caetano EB, Caetano MF, Fregona LR, Neri IDO, Nunes RPS, Campos DLP. Variações do nervo mediano no túnel do carpo. Rev Bras Ortop 2005;40(10):608-613

21 Lanz U. Anatomical variations of the median nerve in the carpal tunnel. J Hand Surg Am 1977;2(01):44-53

23 Tountas CP, Bihrle DM, MacDonald CJ, Bergman RA. Variations of the median nerve in the carpal canal. J Hand Surg Am 1987;12(5 Pt 1):708-712

24 Ragoowansi R, Adeniran A, Moss AL. Anomalous muscle of the wrist. Clin Anat 2002;15(05):363-365

25 Hollevoet N, Barbaix E, D'herde K, Vanhove W, Verdonk R. Muscle fibres crossing the line of incision used in carpal tunnel decompression. J Hand Surg Eur Vol 2010;35(02):115-119

26 Tuncali D, Barutcu AY, Terzioglu A, Aslan G. Transverse carpal muscle in association with carpal tunnel syndrome: report of three cases. Clin Anat 2005;18(04):308-312

27 Henry BM, Zwinczewska H, Roy J, et al. The Prevalence of Anatomical Variations of the Median Nerve in the Carpal Tunnel: A Systematic Review and Meta-Analysis. PLoS One 2015;10(08): e0136477Erratum in: PLoS One 2015;10(9):e0138300 